\title{
Ketosis as a treatment for multiple metabolic and neurodegenerative pathologies
}

\author{
Raffaele Pilla* \\ External Pharmacy of Saint John of God - Fatebenefratelli Hospital, Viale Principe di Napoli 14/B, Benevento, Italy
}

\section{Introduction}

\section{Efficacy of metabolic therapies in animal models of seizure}

It is renown that seizures may be caused by genetic or environmental factors. Central nervous system oxygen toxicity (CNS-OT), also known as hyperbaric oxygen ( $\mathrm{HBO} 2)$-related seizures, compromise the safety of undersea divers using rebreathers and patients undergoing $\mathrm{HBO} 2$ therapy [1]. Breathing $100 \% \mathrm{O} 2$ at the barometric pressure $(\mathrm{Pb})$ of 2.4 atmospheres absolute (ATA), or higher doses, increases the risk of convulsions in exposed subjects, and current applications of $\mathrm{HBO} 2$ therapy routinely use up to $3 \mathrm{ATA} \mathrm{HBO} 2$ [2], which is why CNS-OT currently represents the primary limiting factor in $\mathrm{HBO} 2$ therapy. For this reason, $\mathrm{HBO} 2$ provides a unique, reversible, and reproducible stimulus for generalized tonic-clonic seizures in animal models and is thus an effective model for assessing anti-seizure strategies.

In the past, it has been demonstrated that fasting delays the onset of CNS-OT [3], by shifting brain energy metabolism. For example, fasting for 24 to 36 hours delays the latency time to CNS-OT seizure up to $300 \%$, which is comparable to high doses of anti-epileptic drugs (AEDs) [4,5] and experimental excitatory glutamatergic neurotransmission blockers [6]. Esters of betaxydroxybutyrate (BHB) or acetoacetate (AcAc) can effectively induce a rapid and sustained ketosis [7,8], therefore D'Agostino and colleagues [9] synthesized and tested a ketone ester [R,S-1,3-butanediol acetoacetate diester (BD-AcAc2)], which has been shown to induce therapeutic ketosis in dogs $[10,11]$ and pigs [8] and was proposed as a metabolic therapy for parenteral and enteral nutrition [7]. The group was interested in esters of AcAc because precursors to BHB do not prevent CNS-OT [12], and animal studies suggest that AcAc and acetone have the greatest anticonvulsant potential [13-16]. Authors demonstrated that a single oral administration of the ketone ester BD-AcAc2 caused rapid and sustained elevations of $\mathrm{BHB}(>3$ $\mathrm{mM}), \operatorname{AcAc}(>3 \mathrm{mM})$, and acetone $(\sim 0.7 \mathrm{mM})$ and increased resistance to seizures ( $\mathrm{LS}=574 \pm 115 \%, \mathrm{P}<0.001$ ), compared with control (water) or BD, even though BD caused a significant increase in BHB (Figure 2) [9]. Ketone ester administration produces "fasting ketosis", which effect is well documented in humans and animal models and correlates with a rise in blood ketones $[13,16]$. Dietary-induced hepatic ketogenesis is dependent on maintaining a low insulin-to-glucagon ratio, which quickly reverses with carbohydrate consumption. Seizure protection is also reversed upon ingestion of calories from carbohydrate or excess protein $(>20 \%)$. BD-AcAc2 has little or no effect on blood glucose [9], and thus makes it an attractive option for mitigating CNS-OT and may represent a sought-after strategy for epilepsy to circumvent issues with compliance associated with KD [17]. In addition, D'Agostino and collaborators [9] demonstrated that the anticonvulsant benefits of fasting ketosis are conferred with BD-AcAc2, even during a standard diet ad libitum, reaching higher blood ketones levels than those typically reported in rats fasted

24-36 $\mathrm{h}[12]$ or rats eating a $\mathrm{KD}[18,19]$. In fact, measurements of $\mathrm{BHB}, \mathrm{AcAc}$, and acetone together showed values of $\sim 6 \mathrm{mM}$, usually achieved in a week of fasting for humans [20]. Acetone levels elevated by BD-AcAc2 $(0.7 \mathrm{mM})$ were similar to brain acetone levels in epilepsy patients that have achieved complete seizure control with adherence to a strict $\mathrm{KD}[21]$.

In order to further evaluate the anticonvulsant properties of orally administered ketone bodies, Viggiano and colleagues tested BD-AcAc2 in an adult Winstar rat model of seizures, induced by pentylenetrazole (PTZ) injection [22]. The PTZ model was chosen as pro-convulsing agent as it represents one of the most commonly employed drugs in acute convulsive crisis models, highly comparable to generalized seizures in humans. Moreover, Bough and Eagles demonstrated how efficient a KD can be in PTZ-induced seizures in a rat model [23]. Viggiano and colleagues assessed the blood ketones increase following the oral administration of $\mathrm{BD}-\mathrm{AcAc} 2$ in rats, in parallel with the CNSOT seizure model reported previously [9]. The main preliminary finding from Viggiano's study was that oral administration of BDAcAc2 manifested a significant increase of BHB and an even more consistent resistance against the epileptic seizure onset, compared to the control group [24].

The Ketogenic Diet as a Universally Recognized Alternative Treatment for Refractory and non-Refractory Epilepsy.

In the event of glucose availability restriction, more than $60 \%$ of human brain's energy will be derived from ketones. After prolonged periods of fasting or ketogenic diet (KD), the whole body utilizes energy obtained from free fatty acids (FFAs) released from adipose tissue. However, the brain is not capable to obtain significant energy from FFAs, thus hepatic ketogenesis converts them into ketone bodies: $\beta$-hydroxybutyrate (BHB) and acetoacetate (AcAc), while a percentage of AcAc spontaneously decarboxylates to acetone [20].

To date, it has been broadly demonstrated how the metabolic state of mild ketosis, which can be induced through $\mathrm{KD}$ administration, calorie restriction or fasting, represents an emerging tool for the metabolic management of epilepsy and a number neurodegenerative

Correspondence to: Raffaele Pilla, Pharm.D., Ph.D, External Pharmacy of Saint John of God - Fatebenefratelli Hospital, Viale Principe di Napoli 14/B, Benevento, Italy, E-mail: raf.pilla@gmail.com

Received: November 22, 2017; Accepted: December 15, 2017; Published: December 19, 2017 
disease [25], amyotrophic lateral sclerosis [26], and some types of cancer $[27,28]$. In addition, nutritional treatments represent a valid alternative whereas pharmaceutical approaches have shown a number of failures and ineffectiveness, not to mention the elevated number of side effects and costs for public health to be faced every year worldwide.

Under normal conditions and mostly in western societies, a healthy brain utilizes glucose as primary energy source, which unbalance can lead to a number of neurodegenerative disorders often associated with mitochondrial impairment and glucose transport-related dysfunctions, such as in epilepsy, traumatic brain injury, Parkinson's and Alzheimer's diseases [29-33]. Ketone bodies and Krebs cycle intermediates represent the best fuels for brain and other organs. In fact, through their utilization, impaired glucose metabolism may be bypassed and their neuroprotective properties may be exploited [34].

However, neuroprotective mechanisms of ketosis are currently object of studies as mechanisms of action are still not sufficiently understood. It has been shown that ketone bodies are neuroprotective as they induce a consistent increase in mitochondrial biogenesis regulating the synaptic function, and also generate ATP increases, thus reducing the reactive oxygen species production in neurological tissues [35,36], and notably inhibit superoxide synthesis in primary rat neuronal cultures exposed to hyperoxia $[37,38]$. Moreover, the main reason why the $\mathrm{KD}$ has been proven so effective as an anticonvulsant approach is because it significantly reduces the metabolism of glucose [19]. In addition, Ma and colleagues [39] demonstrated that, at physiological concentrations, $\mathrm{BHB}$ and AcAc reduce spontaneous discharges of GABAergic. neurons in the rat substantia nigra, through ATP-sensitive potassium channels. Also, a reduction of total CNS aspartate levels in association with an increase of glutamate concentrations was found during ketosis, observing a significant increase of decarboxylated glutamate to GABA, the main inhibitory neurotransmitter [40-44]. Moreover, a remarkable increase in mitochondrial transcription enzymes and proteins was observed in rat hippocampus after the administration of a KD [45]. Taken together, findings in the field suggest that neurons may resist to depolarization through ionic gradient and rest potential homeostasis, which might explain the evident analogy between the anticonvulsant mechanisms of orally administered ketone bodies and KD.

Epilepsy represents one of the most frequent neurological pathologies as it concerns about 43 million people worldwide. It results from a variety of CNS disorders and can be determined by vascular damages, genetic factors or malformations, cancers, pre-/post-natal injuries, traumatic brain injury. It has been demonstrated that the $\mathrm{KD}$ is one of the most effective non- pharmacological approaches in refractory epilepsy [46], although it is still unknown to and underestimated by many neurologists. Furthermore, the KD can be associated with classic antiepileptic drugs, thus significantly increasing their therapeutic results [4]. The KD induces a consistent increase in blood ketone concentration, notably AcAc and acetone $[13,16]$ and its full success has been shown in about $50 \%$ of epileptic cases (complete seizure elimination), whereas it still significantly improves the quality of life for the remaining $50 \%$ of patients [16]. Furthermore, it has been reported that ketosis may be beneficial against cancer by decreasing blood glucose levels, the primary metabolic fuel for cancer cells [27,4749]. In fact, previous work highlighted that blood ketone concentration was negatively correlated with tumor growth.

\section{The role of ketones in neurodegenerative diseases}

Impairment of mitochondrial function is most likely the main cause of many neurodegenerative diseases by causing dysfunction in energy production. Metabolic therapies that target mitochondrial function could stall or reverse the progression of neurodegenerative diseases, such as Alzheimer`s disease (AD), amyotrophic lateral sclerosis (ALS), multiple sclerosis and traumatic brain injury (TBI) by preserving neuronal function and decreasing ROS. Ketones may confer a therapeutic effect, especially against neurodegenerative diseases that are characterized by impaired glucose metabolism. Transgenic animal models of neurodegenerative diseases showed positive outcomes induced by ketogenic diet. Increased motor neuron number was reported in ALS transgenic models in response to ketosis (Zhao et al. 2006; Zhao et al. 2012), reduced lesion volume was described after TBI [50], increased cell survival and decreased seizure frequency in kainate-induced seizure models were observed [51] and suppressed inflammatory cytokines and chemokines in an experimental model of multiple sclerosis was reported [52].

\section{Metabolic therapies in mouse models of alzheimer's disease}

Since KD has been proven to improve metabolic efficiency in seizure disorders by acting as an anticonvulsant [13,53-57], it has been suggested that the diet may be useful for treating several other neurological disorders, such as Alzheimer's disease (AD). Especially, since patients affected with $\mathrm{AD}$ often experience seizures [57], while it has been reported that neuronal excitability is enhanced $[58,59]$ and neuronal circuits and mitochondrial homeostasis are altered. $\mathrm{AD}$ manifests as a progressive decline in memory, and affects an estimated 5.4 million people in the US. This number is expected to double by 2050 (Alzheimer's Association, 2012). The main pathological hallmarks of the disease are extracellular deposits of amyloid beta $(A \beta)$, intracellular accumulation of neurofibrillary tangles (tau deposition), and progressive loss of neurons. In $\mathrm{AD}$ patients, similarly to other dementias, an energy imbalance is present. Hypometabolism can be observed in several brain areas, especially in the hippocampus [60,61] as well as impaired mitochondrial function [62]. There is a shift in brain metabolism as an early event in $\mathrm{AD}$ pathology, which results in decreased cerebral glucose utilization, while changing from utilization of glucose to increased ketogenesis during aging [63,64]. Providing ketone bodies as an alternative fuel for neurons has been suggested to bypass such metabolic deficits present in the $\mathrm{AD}$ brain.

Researchers use several transgenic mouse models to elucidate the mechanism of the disease and to test potential treatment options. In an animal model of $\mathrm{AD}$, transgenic mice consuming a ketogenic diet exhibited better mitochondrial function and less oxidative stress and b-amyloid deposition when compared with normally fed controls [65] (Table 1).

Two, well established mouse models with Alzheimer-like pathology have been tested so far [66]. Five months old amyloid-depositing mice, transgenic for mutant amyloid precursor protein and presenilin-

Table 1. Comparison of diabetic ketoacidosis to therapeutic ketosis.

\begin{tabular}{|c|c|c|}
\hline & Diabetic Ketoacidosis & Therapeutic Ketosis \\
\hline Blood Ketones (mM) & $>10-20$ & $0.5-8$ \\
\hline Insulin & Dysregulated/Absent & Low \\
\hline Glycemia & High & Low \\
\hline Renal Metabolism & $\begin{array}{l}\text { Ketonuria, glycosuria, } \\
\text { reduced GFR }\end{array}$ & Mild osmotic diuresis \\
\hline Acidosis & Very high & Mild and regulated \\
\hline Pathology & $\begin{array}{l}\text { Hypovolemia, hypotension } \\
\text { and death }\end{array}$ & None \\
\hline Cognitive Performance & Impaired & Enhanced \\
\hline Physical Performance & Impaired & Enhanced \\
\hline
\end{tabular}


1(APP+PS1) and tau depositing mice transgenic for tau (Tg4510) were fed with low carbohydrate, medium chain triglyceride-rich diet, which induced ketosis, but did so without introducing high amounts of omega- 6 or hydrogenated fats. Medium chain triglycerides were used, since those can be metabolized in the liver rapidly to produce a mild state of ketosis $[67,68]$. The study demonstrated that the ketogenic diet effectively elevated circulating ketone bodies, while successfully reduced glucose levels. The behavioral and histopathological outcomes of this study were less prominent. KD enhanced motor performance in Tg4510 mice, but neither rescued neuronal loss, nor improved memory deficits. During the last two weeks of treatment the mice that were fed with KD performed significantly better on the rotarod, while in the radial arm water maze test learning deficits were identified in both transgenic lines with no significant differences between diets.

Other studies showed similar results; KD improved rotarod performance in young (1-2 month) nontransgenic and APP+PS1 mice when fed KD for one month [69], while no effects on the soluble amyloid in the brain or muscle could be detected by ELISA, similarly to the previous study [66].

The effects of a ketone ester have also been tested in a recent study [70] on a 3xTg mice by reaching $0.7 \mathrm{mM}$ BHB. After 4-7 months of treatment subtle differences in learning were observed, with decreased anxiety and increased locomotor activity in the ketone- supplemented mice. These transgenic mice do not have amyloid deposits, but they exhibit intracellular staining with an anti- $\mathrm{A} \beta$ antiserum. Interestingly, in the ketone ester supplemented group the number of cells exhibiting this staining was reduced.

In a female $\mathrm{AD}$ mouse model reduced mitochondrial APP and the $16 \mathrm{kD}$ Abeta oligomer levels were reported after treatment with 2-deoxyglucose (2-DG) [64]. 2-DG competitively blocks glucose metabolism; therefore, it induces a compensatory rise of ketone bodies by the liver [71] which might have led to the neuroprotective effect.

While some of these results are encouraging, it is necessary to further improve our understanding about the potential benefits of KDs in $\mathrm{AD}$, which still remains an irreversible disease.

\section{Metabolic therapies in mouse models of ALS}

Currently, the most prevalent motor neuron disease is ALS, with a lifetime risk of 1 in 2000 [72]. While the mechanism of the disease remains elusive, it results in neuronal death characterized by excess glutamate and oxidative stress-induced metabolic dysfunction. The exact cause of ALS is still unknown, proteinopathy, glutaminergic dysregulation, metabolic dysregulation and motor neuron death all occur in ALS and suggest various neuroprotectants as possible therapy. Hallmarks of ALS also include mitochondrial dysfunction, oxidative stress, glutamate excitotoxicity, and ultimately death of motor neurons $[73,74]$. To date, the only therapy offered to ALS patients to extend survival is riluzole, which offers only a modest (2-3 months) extension of survival in some patients, and has considerable side effects [75].

In order to understand the mechanism of the disease and to test different therapeutic approaches, transgenic ALS mouse models are often utilized model organisms. The most commonly used transgenic mouse model is the SOD1-G34A line. These transgenic mice express mutant forms of the human SOD1 gene and multiple copies of the wild type (wt) human SOD1 gene, therefore, providing the opportunity to study the progression of ALS pathogenesis [76,77]. Recent studies suggest that the above mentioned histopathological and biochemical hallmarks result from impaired energy metabolism. Scientists tested the ketogenic diet in SOD1-G34A mice to restore energy balance as a collateral metabolic therapeutic approach. Zhao et al. [78] reported both histological and functional improvements when the transgenic mice were fed by the ketogenic diet compared with a control diet. The study described improvement in motor function as well as higher motor neuron count; however, the survival time was not increased.

In addition, Zhao et al. [79] has shown that caprylic triglyceride, which is a medium chain triglyceride, stalled the impairment of motor function and reduced the death of motor neurons in the spinal cord in the transgenic ALS mice through the restoration of energy metabolism. The survival time was not increased in this study either, the ketogenic diet and caprylic triglyceride were each shown to provide alternative fuel, but could not achieve extended survival. From these results it is clear that elevated blood ketone level enhances motor performance, but is not sufficient for extending survival.

Studies on ALS transgenic mice are crucial to test potential therapies that not only improve motor function, but extend survival, especially if anecdotal reports in humans suggest a therapeutic effect. Anecdotal reports from ALS patients (Winning the Fight against Neurodegenerative Diseases Foundation) describe symptomatic improvement in motor control following administration of the supplement component of the Deanna protocol (DP). The DP is comprised of arginine alpha-ketoglutarate (AAKG) as the main ingredient and a number of other agents that were reported to preserve metabolic function and prevent glutamate excitotoxicity associated with ALS-induced metabolic dysfunction. The DP was developed to slow the disease progression which is caused by the premature death of motor neurons by using the metabolic intermediate AAKG in combination with mitochondrial ATP production supporting agents, through supplying an alternative energy source for the neurons and glial cells [26].

A recent study on SOD1-G34A mice tested the DP supplement as a potential metabolic therapy. The animals were randomly assigned to one of four groups: Standard Diet (SD; Control); SD + Metabolic Therapy (SD+DP); Ketogenic Diet (KD); or KD+DP $(n=11$ each group). The DP was composed of AAKG (arginine alpha-ketoglutarate in 1:1 ratio, Primaforce, 10\%); GABA (gamma-aminobutyric acid, Primaforce, 1\%); Bio-enhanced ubiquinone (HQ2, Tishcon, $0.1 \%$ ); medium chain triglyceride (MCT) high in caprylic acid (MCT oil, Life enhancement, 10\%) [26].

Mice fed with ketogenic diet or the Deanna Protocol (DP) supplementation showed improved motor performance similar to what was found in previous studies [78,79]. KD-fed mice exhibited better motor performance on accelerating rotarod, grip test and hanging wire motor function tests between 15 and 16 weeks of age. The DP supplementation added to a standard rodent diet improved motor function, delayed neurological deficits, and significantly extended survival in SOD1-G93A mouse model of ALS. The major findings of the study show that during the later stages of the disease progression the $\mathrm{SD}+\mathrm{DP}$ mice demonstrated improved motor function on rotarod, grip test, and PAGE test as well. SD+DP group exhibited a significantly better neurological score from age 116 to 136 days. In addition, $63.3 \%$ and $72.7 \%$ of animals lived past 125 days in $\mathrm{KD}+\mathrm{DP}$ and $\mathrm{SD}+\mathrm{DP}$ groups, respectively, while only $9 \%$ of the control animals remained alive past that time point. Therefore, it can be concluded that metabolic therapy using DP supplement delayed disease progression and extended survival in the SOD1-G93A mouse model [26].

A recent study on SOD1-G34A mice describes extended survival in response to resveratrol as well [80]. Resveratrol is considered an 
anti-aging antioxidant that is found in many foods such as red wine, peanuts and Japanese knotweed (Polygonum cuspidatum). It has been found to suppress the influx of excitatory ions into some cell types which is associated with reduced glutamate-induced cell toxicity [81]. Resveratrol may target neurodegenerative diseases by reducing oxidative stress, both on its own and by increasing the expression of SIRT1 [82], a gene associated with longevity. In addition, resveratrol has been reported to increase the activity of SOD in cells and therefore protect them from apoptosis and oxidative stress [83], thus preserving metabolic function even in the presence of a persistent molecular pathology.

In the study by Mancuso et al. [80] resveratrol treatment from 8 weeks of age significantly delayed disease onset and preserved lower and upper motorneuron function in the SOD1-G34A mouse model. In addition, it extended the lifespan of the transgenic mice and promoted survival of their spinal motorneurons. It is speculated that resveratrol's protective effects were associated with increased expression and activation of Sirtuin 1 and AMPK in the ventral spinal cord, which mediators increased mitochondrial biogenesis and ultimately enhances cellular bioenergetic potential.

These studies suggest that targeting energy metabolism with metabolic therapy produces a therapeutic effect in ALS mice and is a feasible strategy that may prolong survival and quality of life in ALS patients. It is also important to mention that presently there are no clinical trials underway to test such metabolic therapies, so it is problematic to extrapolate the potential role of such therapies in humans.

\section{Metabolic supplementation}

Ketone supplementation would circumvent the need for dietary restriction that is required to sustain nutritional ketosis. For this reason, ketone supplementation and other forms of energy intermediates are being developed and tested as forms of metabolic therapy. Ketogenic medical foods or exogenous ketones have proven problematic for reasons of cost, palpability, tolerability and longterm safety. Ketogenic fats, like medium chain triglyceride oil (MCT oil) are poorly tolerated by the gastrointestinal system, but studies have shown that cognitive enhancement benefits can be observed from the mild ketosis in those individuals tolerant to MCT-based formulas [32]. Oral administration of BHB and AcAc in their free acid form is expensive and ineffective at producing sustained ketosis. The sodium salt of BHB has been shown to elevate blood ketone levels in animal models [13], while esters of $\mathrm{BHB}$ or AcAc also promise great therapeutic potential $[8,9,36]$. These ketone esters of BHB are demonstrated to be safe and well tolerated in rats [84] and humans [85], while they produce fasting-level ketosis without dietary restriction. Ketone esters may represent the soughtafter "ketogenic diet in a pill" [17], but further testing of these ketogenic agents are required.

In addition, a novel nutritional approach in immunotherapy of cancer based on ketogenic diet supplemented with chondroitin sulfate, vitamin D3, oleic acid and fermented milk showed the efficacy of a newly commercialized line of non-glucidic food products utilized in cancer patients' daily diet (Dolce Vita LLC-Gilbert, AZ, USA) [86]. This new clinical supplementation might represent the future in terms of ketogenic nutrition for the treatment of pathologies such as cancer, obesity, diabetes type II, refractory and non-refractory epilepsy and a number of neurodegenerative diseases.

\section{Potential future application}

Nutritional therapies are increasing in popularity and are in the main focus in other neurological diseases as well. The possible beneficial effects of ketogenic diets on mitochondrial activity might explain the reports on improvement of patients' scores in Parkinson' disease [87]. Use of $\mathrm{KD}$ has also been shown to reduce the volume of cortical contusion in an animal model of cortical injury [50]. Since TBI may lead to epilepsy in some cases and because of the effective use of the ketogenic diet in reducing seizures, it has been suggested that it may also improve the clinical status following TBI. Ketone supplements are being developed, medical foods and dietary supplements are emerging in order to help keep low blood glucose levels and elevate ketone levels without dietary restrictions.

Future research will need to target the question whether Parkinson disease, glioblastoma, TBI and other metabolic disorders, such as Friedreich's ataxia, Multiple Acyl-CoA Dehydrogenase Deficiency (MADD), PDH deficiency, GLUT1DS can be alternatively treated with similar metabolic therapies.

\section{Concerns}

Previously, ketones were considered as "metabolic poison" and there has been much confusion about the physiological state of nutritional ketosis in the medical community [29]. Many of these concerns result from the association of therapeutic ketosis with diabetic ketoacidosis (DKA). DKA produces "runaway ketosis" and results in ketone concentrations of $20 \mathrm{mM}$ or greater, which can be reversed with insulin administration. A major concern that frequently arises with regard to ketosis is related to the observation that blood $\mathrm{pH}$ may transiently decrease during the initial stages of ketosis [88] caused by the accumulation of ketone bodies in the bloodstream. However, several studies have demonstrated $[8,10,11]$ that the mild $\mathrm{H}+$ load and blood $\mathrm{pH}$ typically return to the normal range as long as ketones are maintained below $10 \mathrm{mM}$ [88].

\section{References}

1. Clark JM, Thom SR (1997) Toxicity of oxygen, carbon dioxide, and carbon monoxide. In: Bove AA, editor. Bove and Davis' Diving Medicine. 3rd ed. Philadelphia, PA: Saunders. 131-145.

2. Tibbles PM, Edelsberg JS (1996) Hyperbaric-oxygen therapy. N Engl J Med 334: 1642 1648 .

3. Bitterman N, Skapa E, Gutterman A (1997) Starvation and dehydration attenuate CNS oxygen toxicity in rats. Brain Res 761: 146-150.

4. Bitterman N, Katz A (1987) The effect of sodium phenytoin on central nervous system oxygen toxicity. Aviat Space Environ Med 58: 224-226.

5. Tzuk-Shina T, Bitterman N, Harel D (1991) The effect of vigabatrin on central nervous system oxygen toxicity in rats. Eur J Pharmacol 202: 171-175.

6. Chavko M, Braisted JC, Harabin AL (1998) Effect of MK-801 on seizures induced by exposure to hyperbaric oxygen: comparison with AP-7. Toxicol Appl Pharmacol 151: $222-228$

7. Brunengraber H (1997) Potential of ketone body esters for parenteral and oral nutrition. Nutrition 13: 233-235.

8. Desrochers S, Dubreuil P, Brunet J, Jette M, David F, et al. (1995) Metabolism of $(\mathrm{R}, \mathrm{S})-1,3$-butanediol acetoacetate esters, potential parenteral and enteral nutrients in conscious pigs. Am J Physiol 268: E660-667.

9. D'Agostino DP, Pilla R, Held HE, Landon CS, Puchowicz M, et al. (2013) Therapeutic ketosis with ketone ester delays central nervous system oxygen toxicity seizures in rats Am J Physiol Regul Integr Comp Physiol 304: R829-836.

10. Ciraolo ST, Previs SF, Fernandez CA, Agarwal KC, David F, et al. (1995) Model of extreme hypoglycemia in dogs made ketotic with (R,S)-1,3-butanediol acetoacetate esters. Am J Physiol 269: E67-75.

11. Puchowicz MA, Smith CL, Bomont C, Koshy J, David F, et al. (2000) Dog model of therapeutic ketosis induced by oral administration of R,S-1,3-butanediol diacetoacetate. J Nutr Biochem 11: 281-287. 
12. Chavko M, Braisted JC, Harabin AL (1999) Attenuation of brain hyperbaric oxygen toxicity by fasting is not related to ketosis. Undersea Hyperb Med 26: 99-103.

13. Bough KJ, Rho JM (2007) Anticonvulsant mechanisms of the ketogenic diet. Epilepsia 48: 43-58.

14. Gasior M, French A, Joy MT, Tang RS, Hartman AL, et al. (2007) The anticonvulsant activity of acetone, the major ketone body in the ketogenic diet, is not dependent on its metabolites acetol, 1,2-propanediol, methylglyoxal, or pyruvic acid. Epilepsia 48: 793-800.

15. Likhodii SS, Serbanescu I, Cortez MA, Murphy P, Snead OC, et al. (2003) Anticonvulsant properties of acetone, a brain ketone elevated by the ketogenic diet. Ann Neurol 54: 219-226.

16. McNally MA, Hartman AL (2012) Ketone bodies in epilepsy. J Neurochem 121: 28-35.

17. Rho JM, Sankar R (2008) The ketogenic diet in a pill: is this possible? Epilepsia 49: 127-133.

18. Bielohuby M, Menhofer D, Kirchner H, Stoehr BJ, Muller TD, et al. (2011) Induction of ketosis in rats fed low-carbohydrate, high-fat diets depends on the relative abundance of dietary fat and protein. Am J Physiol Endocrinol Metab 300: E65-E76.

19. Bough KJ, Gudi K, Han FT, Rathod AH, Eagles DA (2002) An anticonvulsant profile of the ketogenic diet in the rat. Epilepsy Res 50: 313-325.

20. Cahill GF (2006) Fuel metabolism in starvation. Annu Rev Nutr 26: 1-22.

21. Seymour KJ, Bluml S, Sutherling J, Sutherling W, Ross BD (1999) Identification of cerebral acetone by $1 \mathrm{H}-\mathrm{MRS}$ in patients with epilepsy controlled by ketogenic diet. MAGMA 8: 33-42.

22. Viggiano A, Pilla R, Arnold P, Monda M, D'Agostino D, et al. (2015) Anticonvulsant properties of an oral ketone ester in a pentylenetetrazole-model of seizure. Brain Res 1618: 50-54.

23. Bough KJ, Eagles DA (1999) A ketogenic diet increases the resistance to pentylenetetrazole-induced seizures in the rat. Epilepsia 40: 138-143.

24. Viggiano A, Pilla R, Arnold P, Monda M, D'Agostino D, et al. (2016) Different calorie restriction treatments have similar anti-seizure efficacy. Seizure 35: 45-49.

25. Hartman AL, Stafstrom CE (2013) Harnessing the power of metabolism for seizure prevention: focus on dietary treatments. Epilepsy Behav 26: 266-272.

26. Ari C, Poff AM, Held HE, Landon CS, Goldhagen CR, et al. (2014) Metabolic therapy with Deanna Protocol supplementation delays disease progression and extends survival in amyotrophic lateral sclerosis (ALS) mouse model. PLoS One 9: e103526.

27. Poff AM, Ward N, Seyfried TN, Arnold P, D'Agostino DP (2015) Non-Toxic Metabolic Management of Metastatic Cancer in VM Mice: Novel Combination of Ketogenic Diet, Ketone Supplementation, and Hyperbaric Oxygen Therapy. PLoS One 10: e127407.

28. Schwartz L, Seyfried T, Alfarouk KO, Da Veiga Moreira J, Fais S (2017) Out of Warburg effect: An effective cancer treatment targeting the tumor specific metabolism and dysregulated pH. Semin Cancer Biol 43: 134-138.

29. VanItallie TB, Nufert TH (2003) Ketones: metabolism's ugly duckling. Nutr Rev 61 327-341.

30. Veech RL, Chance B, Kashiwaya Y, Lardy HA, Cahill GF, Jr. (2001) Ketone bodies, potential therapeutic uses. IUBMB Life 51: 241-247.

31. Prins ML (2008) Cerebral metabolic adaptation and ketone metabolism after brain injury. J Cereb Blood Flow Metab 28: 1-16.

32. Henderson ST (2008) Ketone bodies as a therapeutic for Alzheimer's disease. Neurotherapeutics 5: 470-480.

33. Hartman AL, Gasior M, Vining EP, Rogawski MA (2007) The neuropharmacology of the ketogenic diet. Pediatr Neurol 36: 281-292.

34. Shimazu T, Hirschey MD, Newman J, He W, Shirakawa K, et al. (2013) Suppression of oxidative stress by beta-hydroxybutyrate, an endogenous histone deacetylase inhibitor. Science 339: 211-214.

35. Maalouf M, Sullivan PG, Davis L, Kim DY, Rho JM (2007) Ketones inhibit mitochondrial production of reactive oxygen species production following glutamate excitotoxicity by increasing NADH oxidation. Neuroscience 145: 256-264.

36. Veech RL (2004) The therapeutic implications of ketone bodies: the effects of ketone bodies in pathological conditions: ketosis, ketogenic diet, redox states, insulin resistance, and mitochondrial metabolism. Prostaglandins Leukot Essent Fatty Acids 70: 309-319.
37. D'Agostino A, Errico ME, Malinconico M, De Rosa M, Avella M, et al. (2011) Development of nanocomposite based on hydroxyethylmethacrylate and functionalized fumed silica: mechanical, chemico-physical and biological characterization. J Mater Sci Mater Med 22: 481-490.

38. Gerschman R, Nye SW, Gilbert DL, Dwyer P, Fenn WO (1954) Studies on oxygen poisoning: protective effect of beta-mercaptoethylamine. Proc Soc Exp Biol Med 85: 75-77.

39. Ma W, Berg J, Yellen G (2007) Ketogenic diet metabolites reduce firing in central neurons by opening K(ATP) channels. $J$ Neurosci 27: 3618-3625.

40. Yudkoff M, Daikhin Y, Horyn O, Nissim I, Nissim I (2008) Ketosis and brain handling of glutamate, glutamine, and GABA. Epilepsia 49: 73-75.

41. Yudkoff M, Daikhin Y, Nissim I, Horyn O, Lazarow A, et al. (2005) Response of brain amino acid metabolism to ketosis. Neurochem Int 47: 119-128.

42. Yudkoff M, Daikhin Y, Nissim I, Horyn O, Luhovyy B, et al. (2005) Brain amino acid requirements and toxicity: the example of leucine. J Nutr 135: 1531S-1538S.

43. Yudkoff M, Daikhin Y, Nissim I, Lazarow A, Nissim I (2001) Brain amino acid metabolism and ketosis. J Neurosci Res 66: 272-281.

44. Yudkoff M, Daikhin Y, Nissim I, Lazarow A, Nissim I (2001) Ketogenic diet, amino acid metabolism, and seizure control. J Neurosci Res 66: 931-940.

45. Bough KJ, Wetherington J, Hassel B, Pare JF, Gawryluk JW, et al. (2006) Mitochondrial biogenesis in the anticonvulsant mechanism of the ketogenic diet. Ann Neurol 60: 223 235

46. Freeman JM, Kossoff EH (2010) Ketosis and the ketogenic diet, 2010: advances in treating epilepsy and other disorders. Adv Pediatr 57: 315-329.

47. Seyfried TN, Kiebish MA, Marsh J, Shelton LM, Huysentruyt LC, et al. (2011) Metabolic management of brain cancer. Biochim Biophys Acta 1807: 577-594.

48. Seyfried TN, Flores RE, Poff AM, D'Agostino DP (2014) Cancer as a metabolic disease: implications for novel therapeutics. Carcinogenesis 35: 515-527.

49. Seyfried TN, Sanderson TM, El-Abbadi MM, McGowan R, Mukherjee P (2003) Role of glucose and ketone bodies in the metabolic control of experimental brain cancer. $\mathrm{Br}$ J Cancer 89: 1375-1382.

50. Prins ML, Fujima LS, Hovda DA (2005) Age-dependent reduction of cortical contusion volume by ketones after traumatic brain injury. J Neurosci Res 82: 413-420.

51. Noh HS, Kim YS, Lee HP, Chung KM, Kim DW, et al. (2003) The protective effect of a ketogenic diet on kainic acid-induced hippocampal cell death in the male ICR mice. Epilepsy Res 53: 119-128.

52. Kim DY, Hao J, Liu R, Turner G, Shi FD, et al. (2012) Inflammation-mediated memory dysfunction and effects of a ketogenic diet in a murine model of multiple sclerosis. PLoS One 7: e35476.

53. Bough K (2008) Energy metabolism as part of the anticonvulsant mechanism of the ketogenic diet. Epilepsia 49: 91-93.

54. Caraballo RH, Vining E (2012) Ketogenic diet. Handb Clin Neurol 108: 783-793.

55. Freeman JM, Kossoff EH, Hartman AL (2007) The ketogenic diet: one decade later. Pediatrics 119: 535-543.

56. Westman EC, Mavropoulos J, Yancy WS, Volek JS (2003) A review of lowcarbohydrate ketogenic diets. Curr Atheroscler Rep 5: 476-483.

57. Palop JJ, Mucke L (2009) Epilepsy and cognitive impairments in Alzheimer disease Arch Neurol 66: 435-440.

58. Noebels J (2011) A perfect storm: Converging paths of epilepsy and Alzheimer's dementia intersect in the hippocampal formation. Epilepsia 52: 39-46.

59. Kapogiannis D, Mattson MP (2011) Disrupted energy metabolism and neuronal circuit dysfunction in cognitive impairment and Alzheimer's disease. Lancet Neurol 10: 187 198

60. NL, Chase TN, Fedio P, Patronas NJ, Brooks RA, et al. (1983) Alzheimer's disease: focal cortical 60 . changes shown by positron emission tomography. Neurology 33: 961965 .

61. Costantini LC, Barr LJ, Vogel JL, Henderson ST (2008) Hypometabolism as a therapeutic target in Alzheimer's disease. BMC Neurosci 9: S16.

62. Blass JP (2000) The mitochondrial spiral. An adequate cause of dementia in the Alzheimer's syndrome. Ann N Y Acad Sci 924: 170-183 
63. Yao J, Hamilton RT, Cadenas E, Brinton RD (2010) Decline in mitochondrial bioenergetics and shift to ketogenic profile in brain during reproductive senescence. Biochim Biophys Acta 1800: 1121-1126.

64. Yao J, Rettberg JR, Klosinski LP, Cadenas E, Brinton RD (2011) Shift in brain metabolism in late onset Alzheimer's disease: implications for biomarkers and therapeutic interventions. Mol Aspects Med 32: 247-257.

65. Van der Auwera I, Wera S, Van Leuven F, Henderson ST (2005) A ketogenic diet reduces amyloid beta 40 and 42 in a mouse model of Alzheimer's disease. Nutr Metab (Lond) 2: 28 .

66. Brownlow ML, Benner L, D'Agostino D, Gordon MN, Morgan D (2013) Ketogenic diet improves motor performance but not cognition in two mouse models of Alzheimer's pathology. PLoS One 8: e75713.

67. Yeh YY, Zee P (1976) Relation of ketosis to metabolic changes induced by acute medium-chain triglyceride feeding in rats. J Nutr 106: 58-67.

68. Liu YM (2008) Medium-chain triglyceride (MCT) ketogenic therapy. Epilepsia 49: 33-36.

69. Beckett TL, Studzinski CM, Keller JN, Paul Murphy M, Niedowicz DM (2013) A ketogenic diet improves motor performance but does not affect beta-amyloid levels in a mouse model of Alzheimer's disease. Brain Res 1505: 61-67.

70. Kashiwaya Y, Bergman C, Lee JH, Wan R, King MT, et al. (2013) A ketone ester diet exhibits anxiolytic and cognition-sparing properties, and lessens amyloid and tau pathologies in a mouse model of Alzheimer's disease. Neurobiol Aging 34: 1530-1539.

71. Patel P (2004) No magic bullets: the ephemeral nature of anesthetic-mediated neuroprotection. Anesthesiology 100: 1049-1051.

72. Gordon PH (2013) Amyotrophic Lateral Sclerosis: An update for 2013 Clinical Features, Pathophysiology, Management and Therapeutic Trials. Aging Dis 4: 295-310.

73. Federico A, Cardaioli E, Da Pozzo P, Formichi P, Gallus GN, et al. (2012) Mitochondria, oxidative stress and neurodegeneration. J Neurol Sci 322: 254-262.

74. Cozzolino M, Carri MT (2012) Mitochondrial dysfunction in ALS. Prog Neurobiol 97: 54-66.

75. Miller RG, Mitchell JD, Lyon M, Moore DH (2007) Riluzole for amyotrophic lateral sclerosis (ALS)/motor neuron disease (MND). Cochrane Database Syst Rev: CD001447.

76. Vinsant S, Mansfield C, Jimenez-Moreno R, Del Gaizo Moore V, Yoshikawa M, et al. (2013) Characterization of early pathogenesis in the SOD1(G93A) mouse model of ALS: part II, results and discussion. Brain Behav 3: 431-457.
77. Vinsant S, Mansfield C, Jimenez-Moreno R, Del Gaizo Moore V, Yoshikawa M, et al. (2013) Characterization of early pathogenesis in the SOD1(G93A) mouse model of ALS: part I, background and methods. Brain Behav 3: 335-350.

78. Zhao Z, Lange DJ, Voustianiouk A, MacGrogan D, Ho L, et al. (2006) A ketogenic diet as a potential novel therapeutic intervention in amyotrophic lateral sclerosis. $B M C$ Neurosci 7: 29.

79. Zhao W, Varghese M, Vempati P, Dzhun A, Cheng A, et al. (2012) Caprylic triglyceride as a novel therapeutic approach to effectively improve the performance and attenuate the symptoms due to the motor neuron loss in ALS disease. PLoS One 7: e49191.

80. Mancuso R, del Valle J, Modol L, Martinez A, Granado-Serrano AB, et al. (2014) Resveratrol improves motoneuron function and extends survival in SOD1(G93A) ALS mice. Neurotherapeutics 11: 419-432.

81. Wu X, Gao J, Yan J, Owyang C, Li Y (2004) Hypothalamus-brain stem circuitry responsible for vagal efferent signaling to the pancreas evoked by hypoglycemia in rat. J Neurophysiol 91: 1734-1747.

82. Sun AY, Wang Q, Simonyi A, Sun GY (2010) Resveratrol as a therapeutic agent for neurodegenerative diseases. Mol Neurobiol 41: 375-383.

83. Yoon DH, Kwon OY, Mang JY, Jung MJ, Kim DY, et al. (2011) Protective potential of resveratrol against oxidative stress and apoptosis in Batten disease lymphoblast cells. Biochem Biophys Res Commun 414: 49-52.

84. Clarke K, Tchabanenko K, Pawlosky R, Carter E, Knight NS, et al. (2012) Oral 28day and developmental toxicity studies of (R)-3-hydroxybutyl (R)-3-hydroxybutyrate. Regul Toxicol Pharmacol 63: 196-208.

85. Clarke K, Tchabanenko K, Pawlosky R, Carter E, Todd King M, et al. (2012) Kinetics, safety and tolerability of (R)-3-hydroxybutyl (R)-3-hydroxybutyrate in healthy adult subjects. Regul Toxicol Pharmacol 63: 401-408.

86. Branca JJ, Pacini S, Ruggiero M (2015) Effects of Pre-Surgical Vitamin D Supplementation and Ketogenic Diet in a Patient with Recurrent Breast Cancer. Anticancer Res 35: 5525-5532.

87. Vanitallie TB, Nonas C, Di Rocco A, Boyar K, Hyams K, et al. (2005) Treatment of Parkinson disease with diet-induced hyperketonemia: a feasibility study. Neurology 64: 728-730.

88. Withrow CD (1980) The ketogenic diet: mechanism of anticonvulsant action. Adv Neurol 27: 635-642.

Copyright: (C2017 Pilla R. This is an open-access article distributed under the terms of the Creative Commons Attribution License, which permits unrestricted use, distribution, and reproduction in any medium, provided the original author and source are credited. 